\title{
ON THE DETERMINATION OF LOOP NATURE IN THE TEM*
}

\author{
M.L. Jenkins \\ Department of Materials \\ University of Oxford, Parks Road \\ Oxford, OX1 3PH, UK \\ 9902452 \\ H. Fukushima \\ Faculty of Engineering \\ Department of Physics and Chemistry \\ Hiroshima University, 1-4-1 Kamiyama \\ 9310950 \\ Higashi-Hiroshima 739, Japan \\ M.A. Kirk \\ Materials Science Division \\ 9700 S. Cass Avenue \\ Argonne National Laboratory \\ Argonne, IL 60439 \\ January 1997
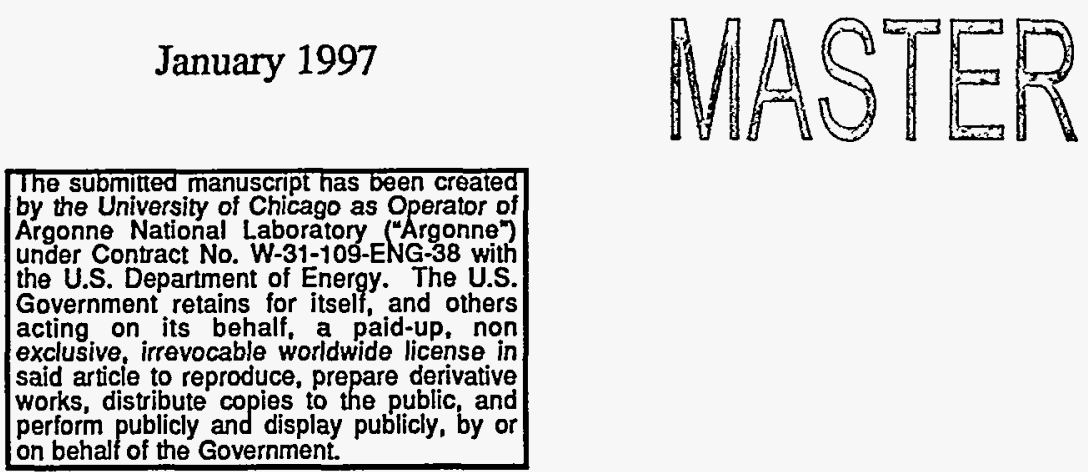 \\ The submitted manuscript has been created
by the University of Chicago as Operator of
Argonne National Laboratory (Argonne")
under Contract No. W-31-109-ENG-38 with
the U.S. Department of Energy. The U.S.
Government retains for itself, and others
acting on its behalf, a paid-up, non
exclusive, irrevocable worldwide license in
said article to reproduce, prepare derivative
works, distribute copies to the public, and
perform publicly and display publicly, by or
on behalt of the Government.

\section{DISCLAIMER}

\begin{abstract}
This report was prepared as an account of work sponsored by an agency of the United States Government. Neither the United States Government nor any agency thereof, nor any of their employees, makes any warranty, express or implied, or assumes any legal liability or responsibility for the accuracy, completeness, or usefulness of any information, apparatus, product, or process disclosed, or represents that its use would not infringe privately owned rights. Reference herein to any specific commercial product, process, or service by trade name, trademark, manufacturer, or otherwise does not necessarily constitute or imply its endorsement, recommendation, or favoring by the United States Government or any agency thereof. The views and opinions of authors expressed herein do not necessarily state or reflect those of the United States Government or any agency thereof.
\end{abstract}

To be submitted to the Materials Research Society Meeting, Boston, MA, December 2-6, 1996.

*Work supported by the U.S. Department of Energy, Basic Energy Sciences-Materials Sciences, under contract \#W-31-109-ENG-38.

NSTRIBUTIOH OF THS DOCUMENT IS UNLIMTED 
DISCLAMMER

Portions of this document may be illegible in electronic image products. Images are produced from the best available original document. 


\title{
M. L. JENKINS", H. FUKUSHIMA“" and M. A. KIRK“”
}

Department of Materials, University of Oxford, Parks Road, Oxford, OX1 3PH, UK "Faculty of Engineering, Hiroshima University, 1-4-1 Kamiyama, Higashi-Hiroshima 739, Japan

..-Materials Science Division, Argonne National Laboratory, Illinois 60439

\begin{abstract}
We have carried out systematic experiments to explore the range of applicability of the two direct methods which are available for the determination of the nature of small clusters. Experiments in heavy-ion irradiated silver and copper, including direct comparisons with the results of Black-White contrast analysis (B-W), forced us to the conclusion that use of the $21 / 2 \mathrm{D}$ technique for the analysis of faulted loops is effectively impossible because of reciprocal-lattice spike and other effects. We used B-W analysis to investigate the nature of the clusters produced by room-temperature heavy-ion irradiation of copper. In-situ irradiations were performed in the Argonne HVEM-Tandem Facility using $40 \mathrm{keV}$ and $80 \mathrm{keV}$ $\mathrm{Kr}^{+}$ions. Nearly all of the analysable first-layer loops were found to be vacancy in nature. Some deeper clusters were also certainly vacancy. There is no unequivocal evidence for interstitial loops under these conditions. The near impossibility of establishing that interstitial loops are not present is shown by the fact that a large fraction of loops - over $50 \%$ - could not be analysed either because they did not display clear B-W contrast under any condition or sufficiently consistent B-W contrast under several different operating conditions.
\end{abstract}

\section{INTRODUCTION}

There is currently considerable interest in the question as to whether resolvable interstitial loops are formed directly within individual displacement collision cascades produced in metals by fast neutron or ion irradiation..$^{1-5}$ However the determination of the nature of small clusters ( $\leq 5 \mathrm{~nm}$ in diameter) is very difficult. In view of these difficulties, and conflicting reports in the literature, we have carried out a systematic experiment to investigate the nature of the clusters produced by room-temperature heavy-ion irradiation of copper and silver using both of the available direct methods: the $2 \frac{1}{2} \mathrm{D}$ technique ${ }^{6}$ and the black-white (B-W) contrast technique ${ }^{7-9}$. The aim of the experiments was to examine critically the range of application of the two techniques, and to assess the evidence for the production of interstitial loops under these irradiation conditions. Full details of the experiments described in outline below will be published in two forthcoming papers. ${ }^{10-11}$

\section{BACKGROUND}

The $2 \frac{1}{2} \mathrm{D}$ technique

The $2 \frac{1}{2}$ D-technique of Mitchell and Bell ${ }^{6}$ attempts to utilise differences in shifts of dark-field images of vacancy and interstitial clusters during defocusing of the objective lens.

The physical principle of the technique is as follows: The elastic strain field causes a local change in the interplanar spacing of the reflecting planes near a loop. This leads to 
an effective local change $\Delta \mathrm{g}$ in the magnitude $\mathrm{g}$ of the operating reciprocal lattice vector. In an out-of-focus dark-field image, obtained with a defocus value of $\Delta D$, the loop image shifts parallel to $\mathrm{g}$ by an amount $\Delta \mathrm{y}=\Delta \mathrm{D} \lambda \Delta \mathrm{g}$ relative to background. Since $\Delta \mathrm{g}$ will have different signs for vacancy and interstitial loops, the images of vacancy and interstitial loops should shift in opposite directions when the focus is changed.

These shift differences are supposed to produce a parallax difference when two (weakbeam) micrographs taken at different focus settings are viewed in a stereo viewer. Weakbeam images are used because of the narrower image widths obtained by this technique. The parallax difference should separate vacancy and interstitial clusters into different "depth" layers. The pseudo-stereo image does not give three-dimensional information about the disposition of the defects in the foil, but neither is it two-dimensional, and hence the name "21/2D-technique".

\section{The B-W contrast technique ${ }^{7-9}$}

Small dislocation loops lying close to either foil surface show so-called black-white (B-W) contrast when imaged under dynamical 2-beam conditions. Calculations show that the sign of the scalar product $\mathrm{g} . \mathbf{l}$ (where $\mathrm{g}$ is the diffraction vector, and $\mathbf{l}$ is a vector from the centre of the black contrast lobe to the centre of the white contrast lobe on a positive print) depends the nature of the loop, and its depth in the foil. The sign of $\mathrm{g.l}$ is opposite for vacancy and interstitial loops in the same depth layer. Near the upper surface the direction of 1 reverses in the second layer L2 (which extends from about $0.35 \xi_{\mathrm{g}}$ to $0.7 \xi_{\mathrm{g}}$, where $\xi_{\mathrm{g}}$ is the extinction distance) compared with the first layer L1 (which extends from the surface to about $0.25 \xi_{\mathrm{g}}$.) In the third layer L3 the contrast is similar to layer L1, although the B-W contrast in this layer may not be very distinct. The situation at the lower foil surface is similar, the only difference being that near this surface bright-field and dark-field images are complementary rather than similar. Loops lying in the transition regions between layers or further than about $1.3 \xi_{\mathrm{g}}$ from a surface are imaged as generally imaged as black dots. The nature of a loop which shows black-white contrast can therefore be determined if it can be placed unequivocally in one of the depth layers. This is usually done by using stereo microscopy to determine the depth of the loop in the foil.

\section{EXPERIMENTAL DETAIIS}

\section{Testing the applicability of the $21 / 2 \mathrm{D}$ technique}

Two experiments were performed. The first was designed to investigate the direction of image shifts produced by defocussing. A true $21 / 2 \mathrm{D}$-effect due to lattice distortions around loops would result in image shifts parallel to $\mathrm{g}$, so that a maximum shift would be measured when the prints are aligned so that $\mathrm{g}$ lies horizontally in the stereo viewer. The same direction and magnitude of image shift would be expected for deviation parameter $s_{g}$ positive or negative for the same defocus values. Any deviation from this behaviour is indicative of image shifts produced by some other mechanism, for example the effect of reciprocal lattice spikes, which will be present if the defect clusters are faulted. Silver specimens were irradiated at room-temperature with $40 \mathrm{keV} \mathrm{Cu}^{+}$ions to a fluence of about $2 \times 10^{15}$ ions $\mathrm{m}^{-2}$. A through-focal series of weak-beam micrographs was recorded using a diffraction vector $\mathbf{g}=\overline{1} 11$ using both positive and negative $s_{\mathrm{g}}$ with the foil oriented close to [110]. Prints were made at a total magnification of $7.5 \times 10^{5}$. Image shifts of pairs of micrographs were then measured with the prints oriented at different angles in the stereo viewer. 
The second experiment was designed to make a direct comparison between the $21 / 2 \mathrm{D}$ technique and the B-W contrast method. In-situ ion irradiations were performed at room temperature in the Argonne HVEM-Tandem Facility. Thin-foil copper specimens were irradiated consecutively with $40 \mathrm{keV}$ and $80 \mathrm{keV} \mathrm{Kr}^{+}$ions to place clusters more uniformly within the top two or three depth layers. As far as possible, B-W and $21 / 2 D$ analyses were then performed on the same defect clusters after each irradiation so that the result of each method could be compared on a defect-by-defect basis. The $21 / 2 \mathrm{D}$ experiment was conducted as described above.

\section{Application of the B-W technique to heavy-ion damage in copper}

In the B-W analysis of the copper specimens irradiated in the HVEM-Tandem, several precautions were introduced in order to improve the reliability of the analysis, and an attempt was made to analyse the whole cluster population, including very small clusters. The layer structure was altered by changing the operating voltage $(100 \mathrm{keV}, 300 \mathrm{keV})$ and operating reflection ( $\mathrm{g}=00 \overline{2}$ and $2 \overline{2} 0$ ). Micrographs were recorded both in-situ in the Kratos HVEM, and after subsequent transfer to a Philips CM30 operating at $300 \mathrm{kV}$. The contrast of several hundred defects was then followed individually under the different imaging conditions. The defects included all those which were visible within the area of analysis. For each reflection, several micrographs taken under slightly different tilt conditions were consulted. The confidence with which the sign of g.l could be assigned was then assessed on a scale from 0 (no assignment possible) to \pm 3 (high confidence). The depth of each defect was measured by the analysis of weak-beam stereo pairs. The depth scale and surface position were confirmed by comparison with computer-generated defect depth distributions using TRMM (modified to yield cascade-centre positions).

Weak-beam microscopy in the Philips CM30 was used to give further geometry information (eg to show the presence of stacking-fault tetrahedra or dissociated Frank loops).

\section{RESULTS}

Results of the $21 / 2 \mathrm{D}$ analyses of defects in ion-irradiated silver

Figures 1 and 2 show the measured image shifts for several clusters (labelled A-1) in ion-irradiated silver as a function of the orientation of the prints in the stereo viewer. Figure 1 shows image shifts of five defects $A-E$ for $s_{g}>0$ (above) and $s_{g}<0$ (below) respectively, while Figure 2 shows the same for a further four defects F-I. The shifts are normalised to a defocus distance of $1 \mu \mathrm{m}$ between each pair of micrographs analysed. The orientation of the prints in the viewer is designated by the angle between the diffraction vector $\mathbf{g}=\overline{1} 11$ and line joining the eyepieces, i.e. $90^{\circ}$ corresponds to placing $g$ so that it lies normal to this line. This would be the orientation where a minimum image shift would be expected if the image shifts are parallel to $\mathrm{g}$.

We note:

(i) Defects A-E in Figure 1 show a minimum image shift at an angle of about $135^{\circ}$ rather than $90^{\circ}$. For a given defect, the slope of the plots reverses with a change in sign of $s_{g}$, but the zero image shift occurs at the same orientation. A detailed analysis shows that this is consistent with image shifts produced by reciprocal lattice spikes arising from stacking faults on inclined $\{111\}$ planes. The positions of the reciprocal lattice spikes and streaks relative to the (111) diffraction spot for the geometry of our experiment are shown (not to scale) in Figure 3 . The shifts in the present case seem 
dominated by spikes $P$ and $Q$. The angle between $r Q$ and $g$ is avoul $1 \mathrm{JJ}$. ine different sense of slope for defects $A-C$ compared with $D$ and $E$ is thought to arise because different spikes are dominant in these two cases: the (111) spike, $P$, for the

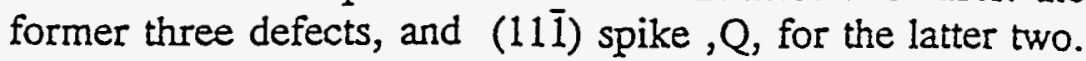

(ii) Defects F-I in Figure 2 show a minimum image shift for an azimuthal angle of $90^{\circ}$ and the same slope for both signs of $s_{g}$. The sense of the slope is consistent with a vacancy nature. However, the experiment described in the next section throws doubt even on this conclusion.

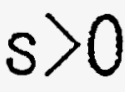

$\{0,0,0\}$

$s<0$

$\{0,0,0\}$
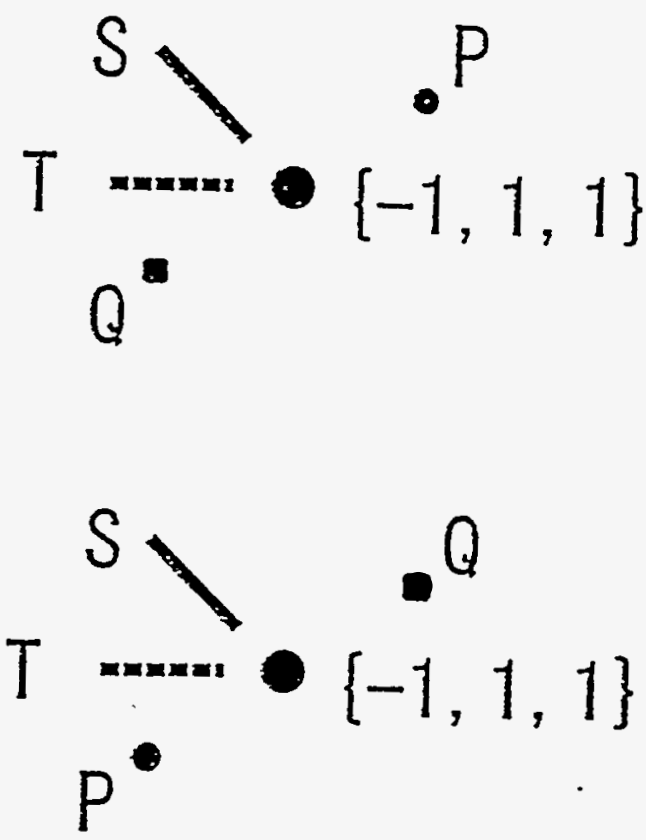

Figure 3 A schematic diagram showing the relative positions of the reciprocal lattice spikes, $\mathrm{P}$ and $\mathrm{Q}$, arising from stacking faults on inclined $\{111)\}$ planes. $\mathrm{P}$ and $Q$ change places with a change in the sign of $s_{\mathrm{s}}$. The streaks, $S$ and $T$, arising from the two edge-on $\{111\}$ planes are also shown. Not to scale.

\section{Results of the $21 / 2 \mathrm{D}$ analyses of defects in ion-irradiated copper}

A defect-by-defect comparison of results obtained by the $2 \frac{1}{2} \mathrm{D}$ technique and the $\mathrm{B}-\mathrm{W}$ contrast technique is summarised in Table $\mathrm{I}$. Defects were designated as "interstitial" or "vacancy" for either technique if their behaviour satisfied certain criteria. For the $2 \frac{1}{2} \mathrm{D}$ technique we required that the minimum image shift occurred at an angle of $90^{\circ}$, and that the slope of the image shift versus orientation plot was similar for both signs of $s_{z}$. If these conditions were not met fully, but the behaviour was not contradictory (if, for example, the 
zero image shift occurred at $90^{\circ}$ for one pair of micrographs, but for the other sign of $\mathrm{s}_{\mathrm{g}}$ image shifts were too small to be measured reliably) then defects were designated "possibly vacancy" or "possibly interstitial". The criteria used for the B-W technique are described below.

Scrutiny of Table I shows that there is no correlation between the results of the two techniques.

\section{The results of the B-W contrast analysis}

Figure 4 shows the results of the $\mathrm{B}-\mathrm{W}$ contrast analysis for four imaging conditions: (a) $\mathbf{g}=00 \overline{2}$, in-situ at $100 \mathrm{kV}$; (b) $\mathbf{g}=00 \overline{2}$, in-situ at $300 \mathrm{kV}$;(c) $\mathbf{g}=002$, ex-situ at $300 \mathrm{kV}$; (c) $\mathrm{g}=2 \overline{2} 0$, in-situ at $300 \mathrm{kV}$. In each case the number of defects with g.l $>0$ is plotted in the positive y-direction, and the number of defects with g.I $<0$ is plotted in the negative $y$-direction. The layer structure for each case is also indicated. The shadings of each part of the columns indicate the confidence with which the g.l assignments could be made. Solid black represents high confidence; diagonal shading medium confidence; and no shading low confidence. It can be seen that most clusters represented by solid black shading lie in the first depth layer for all conditions. Those with g.l $>0$ in the first layer are of vacancy character, and it can be seen that most are of this type. Some second layer vacancy clusters are also evident. This was often corroborated with geometrical evidence: many could be identified as stacking-fault tetrahedra, which almost certainly must be of vacancy type.

Some clusters are present with g.l $<0$ in the first layer, or g.l $>0$ in the second, which is consistent with an interstitial designation. Many but not all of these occur near the change-over region between layers, where the layer allocation is particulary difficult. Some seem to be located far from layer boundaries, however. It is not possible to judge from Figure 4 the strength of the evidence for interstitial clusters, because it is not clear whether the clusters which show interstitial-type contrast are the same in each plot. In order to

Table I:

Comparison of the $2 \frac{1}{2} \mathrm{D}$ result with the $\mathrm{B}-\mathrm{W}$ contrast analysis in $\mathrm{Kr}^{+}$-irradiated $\mathrm{Cu}$

$2^{1 / 2 D}$ result

7 interstitial

9 possible interstitial

4 possible vacancy

21 not analysable
B-W analysis for the same clusters

2 vacancy, 1 possible interstitial, 4 not analysable

3 vacancy, 2 possible vacancy, 4 not analysable

2 vacancy, 1 possible vacancy, 1 not analysable

6 vacancy, 2 possible vacancy, 1 possible interstitial, 12 not analysable

13 vacancy, 5 possible vacancy, 2 possible interstitial, 21 not analysable
TOTAL: $\mathbf{4 1}$ 


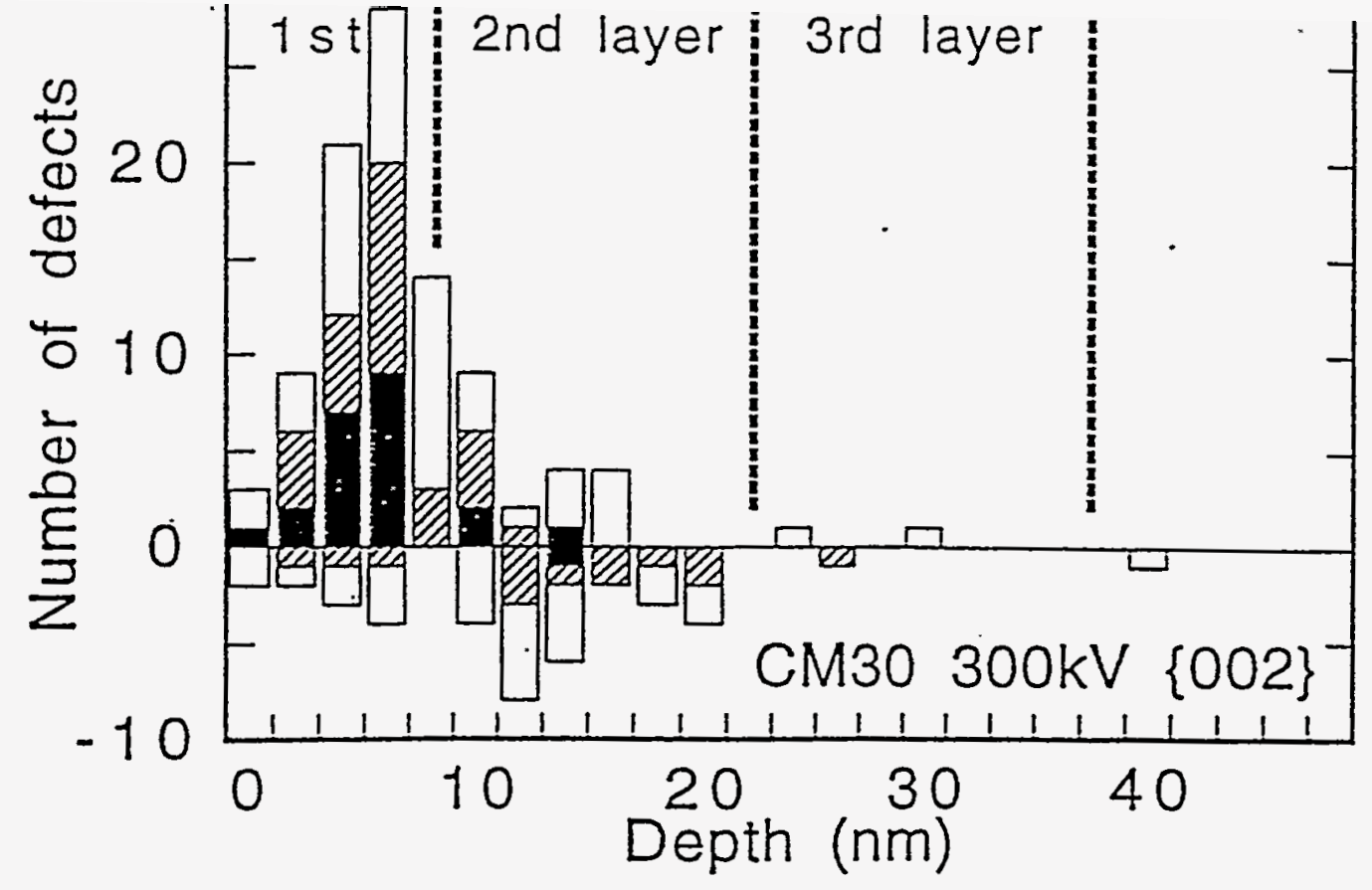

(b)

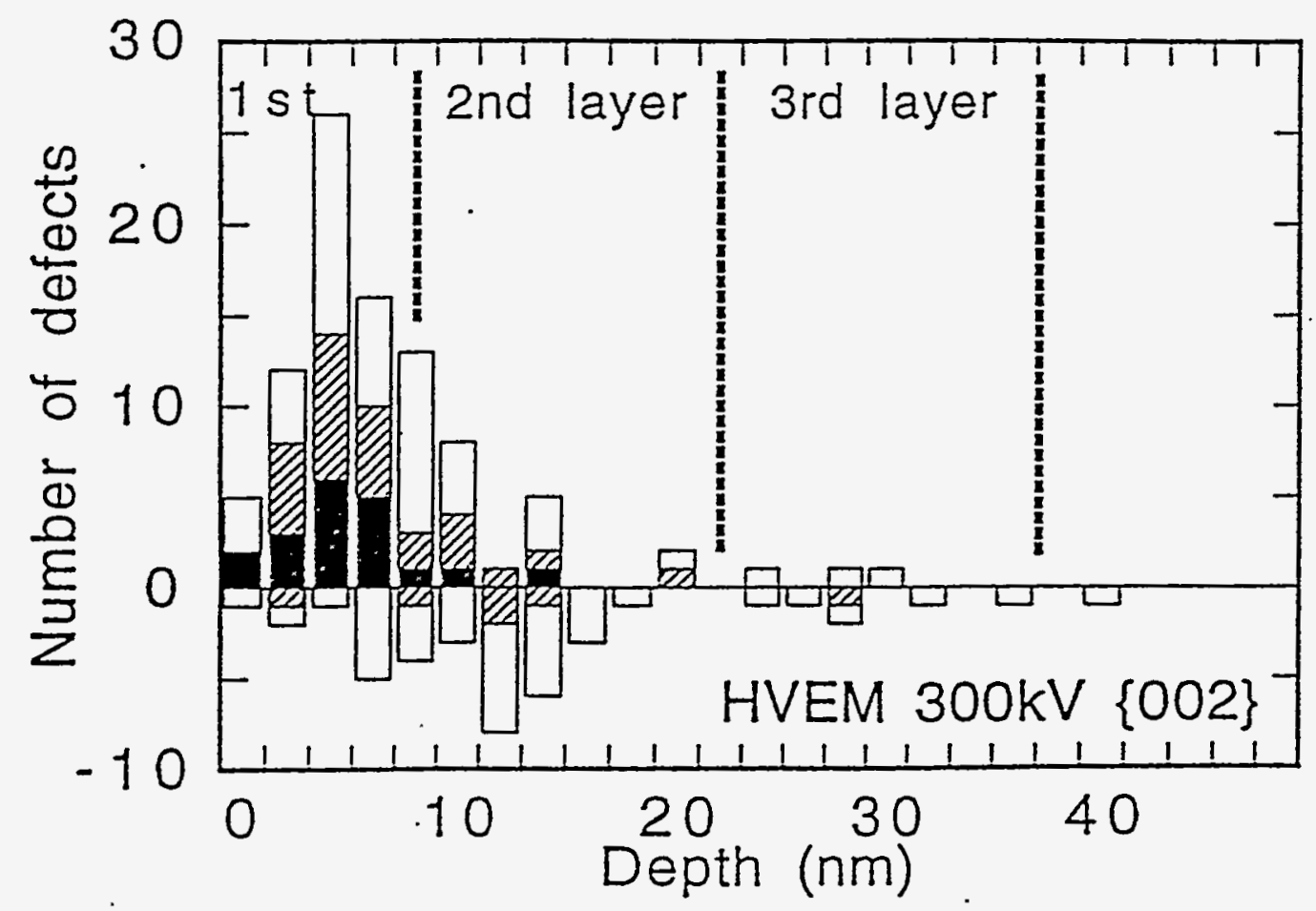

Figure 4 Results of the B-W contrast analysis for four imaging conditions: (a) $\mathrm{g}=00 \overline{2}$, in-situ at $100 \mathrm{kV}$; (b) $\mathrm{g}=002$, in-situ at $300 \mathrm{kV}$ 


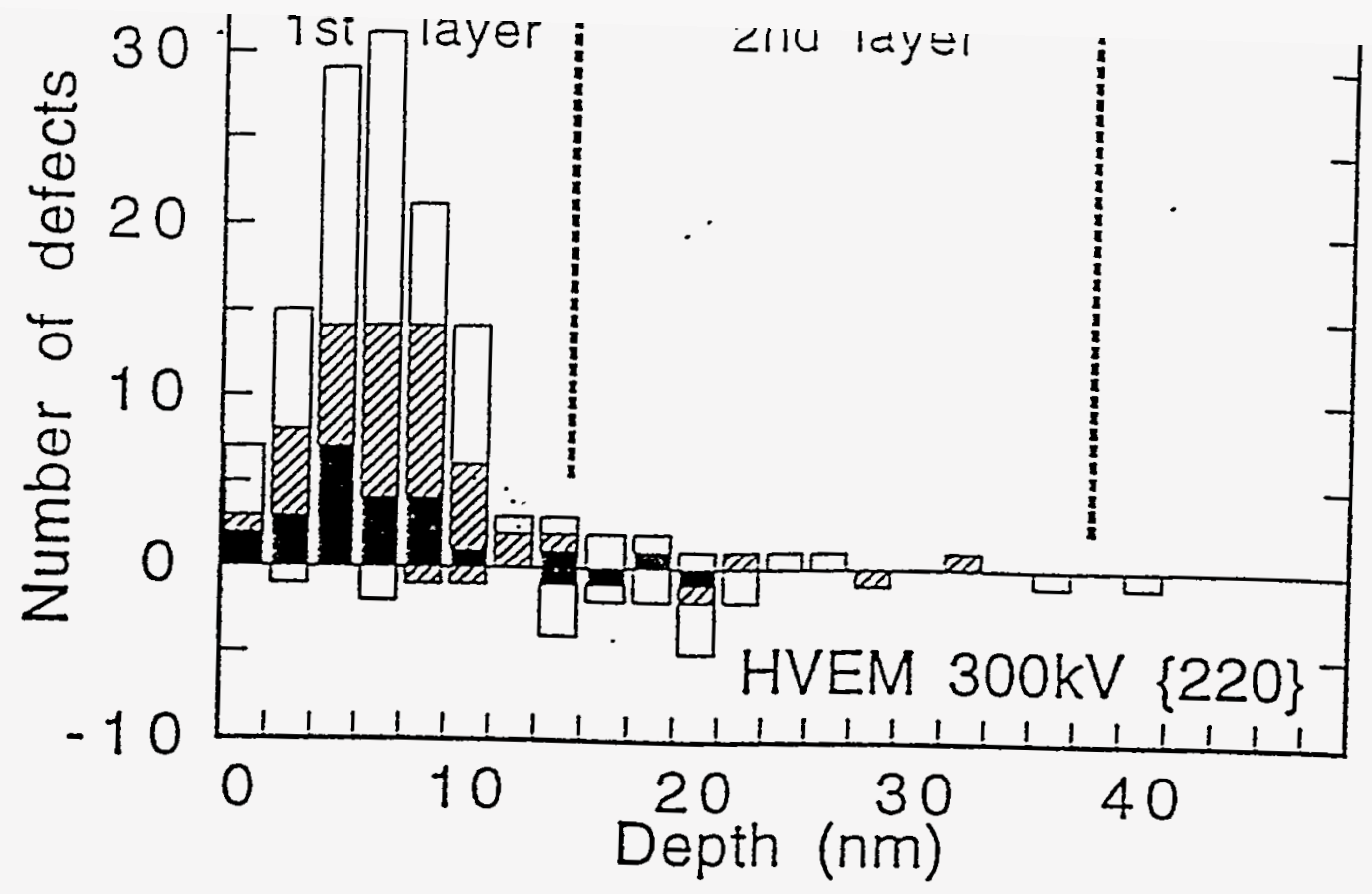

(d)

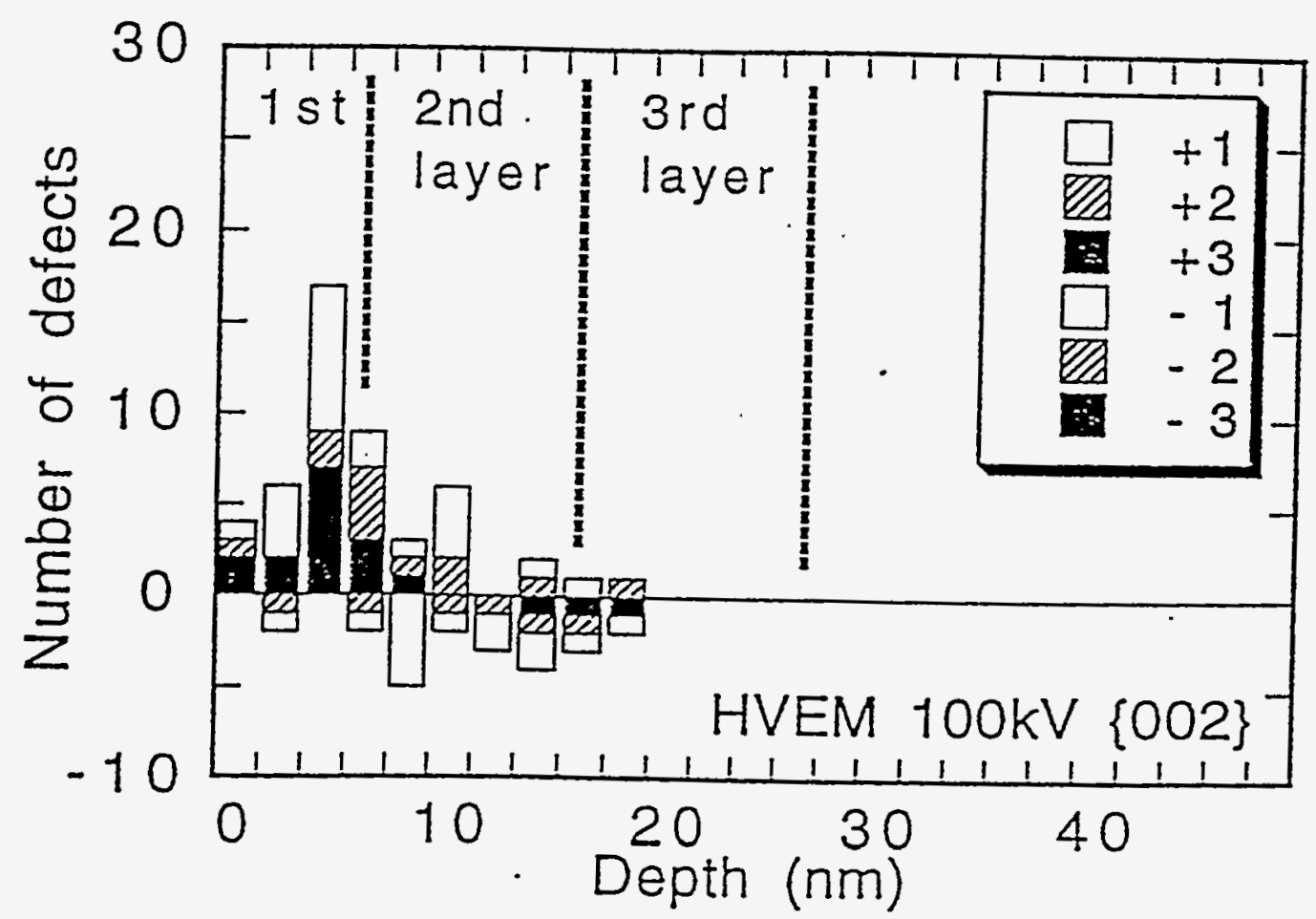

Figure 4 (cont.) Results of the B-W contrast analysis for four imaging conditions: (c) $\mathrm{g}=00 \overline{2}$, ex-situ at $100 \mathrm{kV}$; (d) $\mathrm{g}=2 \overline{2} 0$, in-situ at $300 \mathrm{kV}$.

In each case the number of defects with $\mathrm{g} . \mathrm{I}>0$ is plotted in the positive $y$-direction, and the number of defects with g.I $<0$ is plotted in the negative $y$-direction. The layer structure for each case is also indicated. The shadings of each part of the columns indicate the confidence with which the g.l assignments could be made (see (d) for key.) 
examine if this was the case, it was necessary to examine each defect individually in each of the conditions. It became clear in this analysis that many of the defects which give an interstitial signal in one of the plots of Figure 4 did not show similar interstitial signals in the other plots. The full results of this analysis are shown in Table II.

In this table, defects are recorded as "probably vacancy" or "probably interstitial" only if their B-W contrast was consistent through the analysis set, so that their depth layer assignment could be made with good confidence. Those which gave less clear signals, but which appeared more likely to be interstitial or vacancy on the grounds of their contrast under one or more conditions, and which did not clearly show inconsistent contrast under any condition, were classified as "possibly vacancy" or "possibly interstitial"1. Also shown in the table are the the number of defects which showed clearly inconsistent contrast, and the number which did not show well-developed B-W contrast under any condition.

Examination of Table II shows :

(i) Over $90 \%$ of the analysable clusters are classified as vacancy or possibly vacancy.

(ii) A small number of defects (15) are classified as possibly interstitial, and one only as probably interstitial.

(iii) Over one half (53\%) of the clusters eluded analysis altogether. -

Table II.

Results of the B-W contrast analysis in $\mathrm{Kr}^{+}$irradiated $\mathrm{Cu}$.

Probable vacancy clusters

Possible vacancy clusters

Probable interstitial clusters

Possible interstitial clusters

Inconsistent contrast

No clear B-W contrast total vacancy

total interstitial

65

84

149

1

15

16

15

170

total unanalysable $\mathbf{1 8 5}$

total analysed

350

${ }^{1}$ Full details of how these assignments were made will be given in ref [11] 
The experiments have confirmed that the analysis of small faulted clusters by the $2 \frac{1}{2} \mathrm{D}$ - technique is unreliable. We have found evidence for the existence of reciprocal lattice spike effects. However, even when we analyse only those defects where such effects seem to be absent, there is no agreement with the results of the well-established B-W contrast method. The reasons for this are unclear, but may be related either to experimental difficulties (for example in the choice of markers used to define the reference plane, which are difficult to find) ${ }^{10}$ or to intrinsic problems with the theoretical basis of the technique. Piskunov ${ }^{12}$ has argued that the geometical optics arguement used to justify the basis of the technique is not valid for small stacking-fault tetrahedra and faulted loops. Grüschel and Laupheimer ${ }^{13}$ have similarly concluded that the technique is fundamentally flawed. What is clear is that we have been unable to find conditions where it works successfully. We conclude that results in the literature which depend on use of the $21 / 2 \mathrm{D}$ technique should be treated with extreme caution, and may be entirely wrong.

The difficulties which we encountered in attempting to apply the B-W contrast method to whole cluster populations were more unexpected. The fact that more than half of the defects eluded classification was particulary surprising, and has received little or no previous attention in the literature. In this regard, our attempts to improve the accuracy of the analysis by analysing several sets of micrographs of the same defect population must be judged a failure. However, we note that if (like all previous investigators) we had not taken these precautions but had based our conclusions on the analysis of a single condition only, then we would have found results on defect natures (e.g. the certain presence of interstitial clusters) which our full analysis shows to be unjustified.

The most interesting scientific question which we attempted to address was whether interstitial loops are present under the irradiation conditions used. This question has proven impossible to answer with certainty. We have shown that the majority of clusters, including nearly all of those in the first layer, are vacancy in nature. This result is hardly surprising. It is in accord with the large majority of the literature on room-temperature heavy-ion irradiation of metals. A small fraction of the clusters appeared on the criteria chosen to be more likely to be interstitial than vacancy, but the evidence is weak. We have no unequivocal evidence for the existence of interstitial clusters. However, the large number of unanalysable clusters makes it impossible to exclude the possibility that interstitial clusters are present. We intend to go on to examine conditions where it is perhaps more likely that interstitial loops are produced, in particular irradiation temperatures below stage II, where there is some independent evidence that interstitial clusters are present.

\section{CONCLUSIONS}

1. Use of the $2 \frac{1}{2} \mathrm{D}$ technique for the analysis of faulted loops is effectively impossible because of reciprocal lattice spike and other effects.

2. B-W analysis of clusters in copper produced by energetic $\mathrm{Kr}^{+}$ions showed that nearly all of the analysable first-layer clusters and some deeper clusters were vacancy in nature.

3. A large fraction of clusters - about $50 \%$ - could not be analysed because they did not display clear B-W contrast. 
Ihere was no unequivocal evidence tor interstitial loops under these irradiation conditions. It was impossible however to establish that interstitial loops are not present because so many defects were unanalysable.

\section{ACKNOWLEDGEMENTS}

We thank the staff of the Argonne HVEM-Tandem facility for assistance with the experiments. The work was supported by the U.S. Department of Energy, BES-Materials Sciences, under contract W-31-109-Eng-38

\section{REFERENCES}

1. H. Fukushima, Y. Shimomura and M.W. Guinan, J. Nucl. Mat., 155-157, p.1205 (1988).

2. H. Fukushima, Y. Shimomura, M.W. Guinan and M. Kiritani, Phil.Mag. A60, p.415 (1989).

3. H. Fukushima, Y. Shimomura, M. Kiritani, M. W. Guinan, H. Gerstenberg, I. Mukouda and T. Mitoma, J. Nucl. Mat. 212-215, p.154 (1994).

4. T. D. de la Rubia and M. W. Guinan, Phys. Rev. Lett., 66, p.2766 (1991).

5. A. J. E. Foreman, W. J. Phythian, and C. A. English, Phil. Mag. A66, p.671 (1992).

6. J. B. Mitchell and W. L. Bell, Acta.Met 24 , p.147 (1976).

7. U. Essman and M. Wilkens, phys. stat. sol. 4, p. K53 (1964).

8. M. Rühle, M. Wilkens and U. Essman, phys. stat. sol. 11, p.819 (1965).

9. M. Rühle and M. Wilkens, Phil. Mag.15, p.1075 (1967).

10. H. Fukushima, M. L. Jenkins and M.A. Kirk, Phil. Mag. A, in press (1997a).

11. H. Fukushima, M. L. Jenkins and M.A. Kirk, Phil. Mag. A, in press (1997b).

12. D. I. Piskunov, Sov. Phys. Sol. State, 27, p2125 (1985).

13. M L Jenkins, J. Nucl.Mat. 21, p.124 (1994) quoting University of Stuttgart doctoral theses by W. Grüschel (1979) and A. Laupheimer (1981). 